and development, hypotonia, intellectual disability and seizures. Its frequency is estimated at $1 / 20000$ to $1 / 50000$ births with a female predilection. It's due to a genetic material deletion near the short arm of chromosome 4 (4p-). Through this case report we aim to remind the mains and associated features of this rare disorder as well as its management.

Methods We report the case of male newborn with a WolfHirschhorn syndrome.

Results A full term newborn was born by c-section indicated for a severe growth restriction. Birth weight was $1850 \mathrm{~g}$, length was $45 \mathrm{~cm}$ and head circumference was $30,5 \mathrm{~cm}$. Clinical examination at birth noted an important hypotonia, cyanosis with no associated signs of respiratory distress, distinctive facial features including a broad and flat nasal bridge, a high forehead, widely spaced eyes with iridian coloboma, poorly provided eyebrows, poorly formed ears with a pre-tragic fistula, micrognathia, thin lips and posterior cleft palate associated to a spina bifida occulta, posterior hypospadias, testicular ectopia and mottled skin. Echocardiography showed a type A interruption of the aortic arch. The ultrasound examination of brain and abdomen was normal. The genetic tests concluded to a wolf-Hirschhorn syndrome with a $(4 \mathrm{p})$ deletion. The boy died at the age of one month due to a severe bronchiolitis. Parental genetic tests are planned.

Conclusion Wolf-Hirschhorn is a rare genetic disorder. It may be inherited but generally it occurs as a random event. Its outcome depends on associated malformations especially congenital heart disease. We insist on the importance of genetic counseling and antenatal diagnosis when index cases are registered.

\section{P120 HYPERTENSION AND ACCESSORY RENAL ARTERIES}

${ }^{1}$ Iulia Maria Marina*, 'Orsolya Racz, ${ }^{1}$ Diana Maniu, ${ }^{2}$ Ioana Filimon, ${ }^{2}$ Otilia Fufezan, ${ }^{1}$ Simona Sorana Cainap. ' 2 nd Paediatric Clinic, University of Medicine and Pharmacy 'Iuliu Hatieganu', Cluj-Napoca, Romania; ${ }^{2}$ Radiology Department, Emergency Clinic Hospital for Children, Cluj-Napoca, Romania

\subsection{6/archdischild-2019-epa.475}

Introduction Arterial hypertension is a major risk factor for cardiovascular, cerebrovascular and renal morbidity and mortality. Renovascular disease is the third most common cause of hypertension in children after coarctation of the aorta and parenchymal renal disease. The spectrum of renovascular diseases in children is very broad compared to adults. Besides fibromuscular dysplasia and renal artery stenosis as the main causes, narrow/hypoplastic accessory renal artery or multiple accessory renal artery or multiple renal arteries could also be involved in ischemic nephropathy.

Case report We describe the case of a 15 -year-old girl who presented with palpitations, chest pain and headache. The physical examination revealed a high blood pressure (over the 99th percentile for age, gender and height). She had insignificant personal and family medical history regarding the cardiovascular risk factors. Laboratory analysis and relevant radiological evaluation were obtained in order to determine the etiology for suspected secondary hypertension. Direct plasmatic renin, plasmatic aldosterone, urinary cathecolamines, plasmatic metanephrines, vanilmandelic acid, thyroid function tests were all within normal limits. In order to rule out possible renal artery stenosis, CT angiography of the renal artery was performed, surprisingly demonstrating a small caliber (hypoplastic) accessory right superior renal artery.
Accessory renal artery stenosis/hypoplastic is a possible etiology for secondary hypertension, presumptively leading to renin secretion by the underperfused kidney.

Conclusion Physicians should be alert to the possibility of the causal role of accessory renal arteries in patients with severe and difficult to manage hypertension. For several reasons this possibility may be greater in children.

\section{\begin{tabular}{l|l} 
P121 TO SCAN OR NOT TO SCAN ...AGAIN
\end{tabular}}

Ross Foley ${ }^{*}$, Liam Skinner, Aoife Carroll. University Hospital Waterford, Waterford, Ireland

\subsection{6/archdischild-2019-epa.476}

A 13 year old male was referred to our regional centre for review of a left sided periorbital cellulitis by ENT, ophthalmology and paediatric teams. Left eye pain began 4 days previously and was initially felt to be secondary to allergies. However, one day prior to transfer to our unit intravenous cefotaxime was commenced at referring hospital and neuroimaging (CT Brain) was performed; this revealed a preseptal periorbital cellulitis with no intracranial extension. Upon arrival he was unwell and lethargic and still spiking temperatures whereupon his antimicrobial cover was broadened to include iv flucloxacillin and metronidazole.

Inflammatory markers were elevated with a CRP of 227 $\mathrm{mg} / \mathrm{L}(0-5)$ while white cell count was slightly elevated at $13 \times 10^{9} / \mathrm{L}$ (4-10). Initial blood cultures were negative but repeat blood cultures taken after 3 days of antibiotics were positive with a pathogenic gram negative organism. Following discussion with microbiology colleagues antibiotic coverage was broadened with an initial improvement over 24 hours but subsequently fevers recurred with increasing lethargy but no focal neurological signs. Inflammatory markers were slowly falling. In view of the clinical deterioration repeat imaging was requested, despite a previously normal CT Brain. MRI revealed a subdural abscess anterior to the left frontal lobe and extensive left sided sinusitis. He was emergently transferred for neurosurgical review and urgent surgery to drain the abscess and nasal sinuses. He recovered well following 6 weeks of intravenous antibiotics.

Subdural abscess is a rare but recognised complication of periorbital cellulitis, particularly secondary to sinusitis. A systematic review of the intracranial complications of rhinosinusitis revealed that the vast majority $(70 \%)$ occurred in young adolescent males with a morbidity rate of $27 \%$ and a mortality rate of $3 \%$. In our case repeat neuroimaging was undertaken due to a clinical deterioration and allowed for timely surgical intervention with a good outcome.

\section{P122 CELIAC DISEASE ASSOCIATED WITH DEPRESSION IN ADOLESCENTS}

'Orsolya-Adrienn Rácz* ${ }^{1}$ Georgia Tita, ${ }^{1}$ Alexandru Pârvan, ${ }^{2}$ Elena Predescu, ${ }^{1}$ Simona Căinap. '2nd Pediatric Clinic, University of Medicine and Pharmacy luliu Hatieganu, Cluj-Napoca, Romania; ${ }^{2}$ Child and Adolescent Psychiatry and Addiction Clinic, University of Medicine and Pharmacy luliu Hatieganu, Cluj-Napoca, Romania

\subsection{6/archdischild-2019-epa.477}

Introduction Celiac disease is an immune-mediated systemic disorder elicited by gluten which causes malabsorption and small-bowel mucosal inflammation in genetically susceptible 
individuals. Although the gastrointestinal tract is the target of autoimmune insult, celiac disease is also associated with extraintestinal problems: autoimmune disorders, malignancies, dermatologic conditions, rheumatologist conditions, neurologic and psychiatric disorders. The psychological symptoms are one of the most interesting and unexpected presentations, which may be the result of nutrient malabsorption or increased levels of proinflammatory cytokines.

Case report We present the case of a 15 year old adolescent girl with a history of autoimmune thyroiditis on replacement therapy with levothyroxine, who was hospitalised because of behaviour disorders and oligobradimenorrhea. On clinical examination the patient presented drowsiness, sudden behavioural changes, learning problems, bradylalia and a tendency to isolate herself. Blood tests showed positive anti-TG2 antibodies ( $>10 \mathrm{x}$ normal) and EMA antibodies. HLA typing was also performed and was positive for HLA-DQ2 cis: DQA1*05 - DQB1*02 - DRB1*03. On the psychiatric consult she was diagnosed with a minor depressive syndrome. She was immediately started on a gluten free diet. Although she showed low compliance to the diet on the follow-up examinations, removing gluten resulted in positive effects on the psychiatric symptomatology. We would also like to add that the patient refused the oesophagogastroduodenoscopy and psychological counselling.

Conclusion Recent studies have shown an association between celiac disease and psychiatric conditions, especially depression. Medical personnel should be aware of this atypical presentation of the disease in order to correctly diagnose and treat celiac disease. Also, psychiatric patients who are resistant to traditional therapy should be investigated for celiac disease in case they also present symptoms of $\mathrm{CD}$ or if a positive family history exists.

\section{P123 MATERNAL PERNICIOUS ANAEMIA ASSOCIATED WITH ANOREXIA AND KETOTIC HYPOGLYCAEMIA IN A CHILD WITH METHYLMALONIC ACID AND OROTIC ACID EXCRETION IN THE URINE - A CASE REPORT}

\begin{abstract}
${ }^{1}$ Abhidhamma Kaninde*, ${ }^{2}$ Tracey Conlon, Aida Abedin ${ }^{3},{ }^{4}$ Ingrid Borovickova, ${ }^{5}$ Patricia Fitzsions, ${ }^{6}$ Ina Knerr. ${ }^{1}$ National centre for Inherited Metabolic Disorders, TempleStreet Children's University Hospital , Dublin, Ireland; ${ }^{2}$ National Centre for Inherited metabolic Disorders, Templestreet Childrens University Hospital, Dublin, Ireland; ${ }^{3}$ National Centre for Inherited Metabolic Diseases, Templestreet Children's University Hospital , Dublin , Ireland; ${ }^{4}$ Dept. of Biochemistry, Templestreet Children's University Hospital , Dublin , Ireland; ${ }^{5}$ Dept.of Biochemistry, Templestreet Children's University Hospital , Dublin, Ireland; ${ }^{6}$ National Centre for Inherited Metabolic Disorders, Templestreet Children's University Hospital, Dublin, Ireland
\end{abstract}

\subsection{6/archdischild-2019-epa.478}

Case report A 13 month-old baby girl presented with vomiting and refusal to feed; she was mostly breast feeding. On examination, she appeared off form, underweight, hypotonic and pale. Bedside testing revealed a low blood glucose level of $1.7 \mathrm{mmol} / \mathrm{l}$ with a normal lactate. She was also noted to have mild electrolyte disturbances and a slightly acidotic venous blood gas analysis. Further investigations, including urinary organic acids, revealed marked ketonuria with increased methylmalonic acid (MMA) $(1014 \mu \mathrm{M} / \mathrm{mmol}$ creat., ref. <8) and orotic acid excretion. She was found to be severely deficient in vitamin B12 $(<125 \mathrm{ng} / \mathrm{l})$ which explained her macrocytic anaemia and raised plasma homocysteine (62 microM/l, ref. <8) and urinary MMA. Her initial plasma amino acids showed relatively increased glutamine, low citrulline and arginine with other amino acids relatively low. Plasma ammonia levels were essentially normal. Interventions/Results The patient was initially commenced on intravenous fluids with dextrose and electrolytes. She was also treated with parenteral vitamin B12 injections, along with folate supplements, which caused a marked improvement in mood, tone, and appetite. MMA excretion, homocysteine and blood film normalized although excretion of orotic acid persisted. Further investigations revealed that her mother had low vitamin B12 levels and was positive for parietal cell antibodies, indicative of maternal pernicious anemia. Metabolic work-up also included an Allopurinol load test which was abnormal. Ornithine transcarbamylase (OTC) gene mutation analysis did not detect any pathogenic variants. Although orotic aciduria has a recognized association with megaloblastic anemia, it would not be explained by low serum B12 levels. The family was instructed that their child could still become unwell and would need monitoring and/or dietetic intervention and an emergency regime during periods with increased metabolic stress, such as infections. The girl made a full recovery with no active issues identifiable at $2 \mathrm{y}$ of age.

Conclusion This case report highlights the following points 1) the importance of glucose and ketone measurements along with a full hypoglycemia work up at the point of care, if glucose low, in a child who presents acutely unwell e.g. with poor feeding and vomiting, 2) one should be vigilant for potential maternal causes in an infant especially those who are breastfed, and 3) the potential relevance and diagnostic dilemma caused by incidental findings of yet uncertain significance for this individual.

\section{P124 ARTERIAL ISCHAEMIC STOKE SECONDARY TO VARICELLA VASCULITIS}

Catherine Crowe*. Galway University Hospital, Galway, Ireland

10.1136/archdischild-2019-epa.479

A 5 year old boy presented to the Emergency Department with a 1 day history of evolving right sided hemiparesis and drooling on a background of primary varicella infection 3 months previously. No other significant medical history. No significant family history.

On examination GSC $15 / 15$, vitals were stable. Cardiovascular, respiratory, gastrointestinal, ENT examinations were unremarkable. Neurological examination; speech was normal, on mobilizing hisright leg dragged along floor. No ataxia or foot drop were noted. Cranial nerves II - XII grossly intact. Muscle bulk, tone and reflexes all normal. Reduced power 3/5 in the right upper and lower limbs.

Investigations FBC, U+E, LFT, Coagulation all normal. CSF VZA DNA detected, VZV IgG $>100 \mathrm{mIU} / \mathrm{ml}$.

CT and MRA brain

No abnormality identified. MRI Brain: Acute Left sided ischemic stroke - affecting the left subinsular region and the posterior limb ofthe left internal capsule.

Diagnosis Arterial ischaemic stoke secondary to varicella vasculitis 